\title{
Considérations portant sur les taux croissants de gonorrhée et de gonorrhée résistante aux médicaments : il n'y a pas de temps à perdre
}

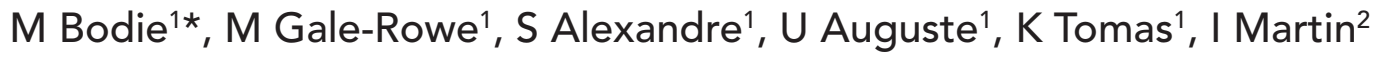

\section{Résumé}

Les taux croissants d'infections gonococciques et de résistance aux agents antimicrobiens observés soulèvent de sérieuses préoccupations en matière de santé publique au Canada et dans le monde entier. Les traitements autrefois recommandés sont devenus inefficaces contre un grand nombre de souches de gonorrhée aujourd'hui omniprésentes. La recommandation actuelle misant sur la thérapie combinée est maintenant menacée par les souches émergentes de provenance internationale qui sont de plus en plus résistantes aux antibiotiques. Il est important de coordonner nos efforts afin d'éviter que ces nouvelles souches mondiales ne s'implantent au Canada; sans quoi nous pourrions voir évoluer ces infections gonococciques persistantes pouvant conduire à la maladie inflammatoire pelvienne, à la stérilité et à la douleur pelvienne chronique chez les femmes et à l'épididymite chez les hommes. La présence d'une infection gonococcique peut aussi accroître le risque de contracter et de transmettre le $\mathrm{VIH}$.

Un certain nombre de raisons peuvent expliquer pourquoi nous faisons face à cette menace à la santé publique. Ces infections gonococciques sont fréquemment asymptomatiques et facilement transmissibles. On peut hésiter à passer un test de dépistage (ou à en proposer un). Le traitement est complexe : les recommandations varient en fonction du site de l'infection et du risque de résistance. Les contacts sexuels à l'occasion de voyages sont une importante source de souches émergentes et résistantes aux antibiotiques importées au pays. Le test d'amplification des acides nucléiques (TAAN), une nouvelle méthode de dépistage et de diagnostic, est excellent, mais il a provoqué une baisse du nombre de cultures produites et, en conséquence, notre capacité de surveillance de la résistance des gonocoques aux agents antimicrobiens s'en est trouvée diminuée.

Les cliniciens et les professionnels de la santé publique de premier plan peuvent adopter quatre grandes mesures pour tenter de freiner l'augmentation des taux d'infection gonorrhéique et de la résistance aux antimicrobiens. Premièrement, la normalisation et l'augmentation du dépistage des infections gonococciques en fonction des facteurs de risque et tout en mettant l'emphase sur l'adoption de pratique sexuelles à risques réduits. Le TAAN est utile au dépistage, mais on a encore besoin de faire des cultures pour les sites extragénitaux. Deuxièmement, prodiguer des conseils aux voyageurs avant leur départ et consigner leurs antécédents de voyages au dossier pour faciliter l'évaluation du risque. Troisièmement, I'utilisation de la culture et du TAAN pour établir le diagnostic et assurer le suivi du contrôle de guérison. Finalement, consulter la version la plus récente des Lignes directrices canadiennes sur les infections transmissibles sexuellement ou des recommandations provinciales ou territoriales en ce qui concerne la thérapie combinée pour les patients et leurs contacts car les recommandations peuvent changer en réponse aux tendances de la résistance des gonocoques aux antimicrobiens qui évoluent.

Citation suggérée: Bodie M, Gale-Rowe M, Alexandre S, Auguste U, Tomas K. et Martin I. Considérations portant sur les taux croissants de gonorrhée et de gonorrhée résistante aux médicaments : il n'y a pas de temps à perdre. Relevé des maladies transmissibles au Canada 2019;45(2/3):58-67. https://doi.org/10.14745/ccdr. v45i23a02f

Mots clés: gonorrhée, gonorrhée multirésistante, ITSS, infection transmissibles sexuellement et par le sang Neisseria gonorrhoeae, résistance antimicrobienne, professionnels de la santé
Cette oeuvre est mise à la disposition selon les termes de la licence internationale Creative Commons Attribution 4.0

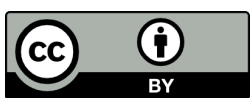

\begin{abstract}
Affiliations
${ }^{1}$ Centre de la lutte contre les maladies transmissibles et les infections, Agence de la santé publique du Canada, Ottawa (Ontario)

2 Division des bactéries pathogènes, Laboratoire national de microbiologie, Agence de la santé publique du Canada, Winnipeg (Manitoba)
\end{abstract}

*Correspondance:
margaret.bodie@canada.ca 


\section{Introduction}

La résistance croissante aux antimicrobiens observée chez Neisseria gonorrhoeae, à la fois à l'échelle nationale et internationale, couplée aux taux croissants d'infections gonococciques, représente une menace sérieuse pour la santé publique. Bien que la gonorrhée soit traitable, les taux mondiaux continuent d'augmenter. L'Organisation mondiale de la Santé estime que 78 millions de personnes sont infectées chaque année par le gonocoque, en raison de l'utilisation décroissante du condom, de l'urbanisation croissante, des voyages plus fréquents, des faibles taux de détection des infections et de l'inadéquation ou de l'échec du traitement (1).

L'Agence de la santé publique du Canada (ASPC) fournit des données annuelles nationales sur les taux d'infections gonococciques (2), la surveillance en laboratoire de la résistance du gonocoque aux agents antimicrobiens (3) et des recommandations sur la prévention, le dépistage précoce et le traitement de la gonorrhée dans Lignes directrices canadiennes sur les infections transmissibles sexuellement (4). En raison de l'augmentation documentée des taux d'infections gonococciques et de la résistance du gonocoque aux agents antimicrobiens, I'ASPC voit la nécessité pour les autorités cliniques et en santé publique de se concerter pour passer à l'action.

Les taux de gonorrhée augmentent de façon constante depuis quelques années. En 2016, le nombre de cas signalés était plus du double de 2010 (étant passé de 33,5 à 65,4 pour 100000 habitants), ce qui correspond à une augmentation de $95 \%$. La population masculine représentait au moins $56 \%$ de tous les cas diagnostiqués. Le groupe d'âge le plus souvent touché était celui des personnes ayant entre 15 et 39 ans; elles représentaient $82 \%$ des cas déclarés de gonorrhée, bien qu'elles ne constituaient que $33 \%$ de la population totale. Les taux ont toutefois doublé pour les personnes de plus de 40 ans depuis les dix dernières années (2).

Actuellement $N$. gonorrhoeae présente une résistance aux six options de traitement autrefois recommandées : les sulfonamides, les pénicillines ainsi que les céphalosporines de première génération, les tétracyclines, les macrolides et les fluoroquinolones (5). Les options actuelles pour le traitement de première intention sont les céphalosporines à spectre élargi (céfixime, ceftriaxone) et l'azithromycine. Afin d'assurer le suivi de la résistance, il faut savoir que la résistance des gonocoques aux agents antimicrobiens s'apparente à deux types : soit les gonocoques multirésistants soit les gonocoques ultrarésistants. Nous définissons les gonocoques multirésistants comme une souche ayant une sensibilité réduite ou une résistance à l'une des thérapies actuellement recommandées (céphalosporine ou azithromycine) plus une résistance à au moins deux autres agents antimicrobiens. Les gonocoques ultrarésistants sont définis comme ayant une sensibilité réduite ou une résistance à deux thérapies actuellement recommandées (céphalosporine et azithromycine) plus une résistance à au moins deux autres agents antimicrobiens $(6,7)$.

Au Canada et ailleurs dans le monde, des isolats affichent des sensibilités réduites aux céphalosporines à spectre étendu et une résistance croissante à l'azithromycine; et des échecs thérapeutiques ont été signalés (8-11). Dans cet article, Martin et al. ont relevé qu'entre 2012 et 2016 , la proportion d'isolats de gonocoques multirésistants a augmenté, passant de 6,2\% à $8,9 \%$ et que celle des gonocoques ultrarésistants était rare $(0,1 \%$ au cours de la même période) (7). Le profil de la résistance variait en fonction de l'antibiotique. La proportion d'isolats ayant une sensibilité réduite aux céphalosporines a diminué entre 2012 et 2016 (passant de 5,9\% à $2 \%$ ) tandis que la résistance à l'azithromycine a augmenté, passant de 0,8 \% à 7,2 \% au cours de la même période avec une certaine variabilité sur le plan régional : les taux étaient supérieurs au Québec (18\%) et en Ontario (5\%) (7).

Tandis que les faibles taux de gonocoques ultrarésistants sont encourageants, les faits concernant les gonocoques multirésistants soulèvent de nouvelles préoccupations au Canada. Depuis l'étude de Martin, on a signalé deux cas liés au voyage concernant des souches de gonocoques résistants à la ceftriaxone n'ayant jamais été observées au Canada. Le premier cas était celui d'une femme asymptomatique dont le partenaire avait voyagé en Chine et en Thaïlande $(11,12)$. Le deuxième cas était celui d'un individu ayant eu des contacts sexuels avec une personne visitant en provenance de l'Asie du Sud-Est. Les isolats des deux cas ont permis d'établir un lien génétique entre la souche FC428 de N. gonorrhoeae, qui a été identifiée pour la première fois au Japon en 2015. Les deux cas ont été traités avec succès : le premier par une dose élevée de céfixime et $d$ 'azithromycine $(11,12)$ et le deuxième par de la gentamicine et de l'azithromycine (communication personnelle, Petra Smyczek, Alberta Health Services, 31 juillet 2018). Cette souche est maintenant identifiée, caractérisée et associée aux voyages dans un certain nombre d'autres pays (Australie, France et Irlande) (11-14). Dans le monde, des cas de gonocoques ultrarésistants résistants à l'azithromycine de haut niveau ont été déclarés au Royaume-Uni et en Australie (15-17); certains de ces cas ont également été associés aux voyages en Asie du Sud-Est.

Il est important de s'efforcer dès maintenant de freiner la progression de la résistance des gonocoques aux antimicrobiens de manière générale et de veiller à ce que les souches dont la résistance est récemment acquise (tant les gonocoques multirésistants que les gonocoques ultrarésistants) ne s'implantent pas au Canada; à défaut de quoi l'important taux de morbidité actuelle associé aux infections gonococciques pourrait connaître une hausse. 
Les infections gonococciques qui n'ont pas fait l'objet d'un diagnostic ou d'un traitement ne sont pas bénignes. Chez les femmes, elles peuvent mener à une infection pelvienne inflammatoire, à une grossesse ectopique, à une douleur pelvienne chronique ou à la stérilité. Chez les hommes, elles peuvent causer l'épididymite (douleur et enflure des testicules). La présence de gonocoques peut également accroître le risque de contracter et de transmettre le VIH (18-20).

Comment cette infection traitable a-t-elle pu devenir une telle menace pour la santé publique? L'objectif de cet article est de déterminer comment cette situation a pu se produire et, ce qui est encore plus important, ce que nous pouvons faire pour y remédier. Nous faisons valoir que chaque clinicien et professionnel de la santé de premier plan peut endiguer la hausse des taux d'infections gonococciques et empêcher de quatre façons les gonocoques multirésistants ou ultrarésistants émergents de s'implanter au Canada.

\section{Comment est-ce arrivé?}

Au moins sept facteurs ont contribué à créer cette situation.

\section{Les infections gonococciques sont souvent asymptomatiques}

Les femmes sont généralement asymptomatiques ou ne présentent que des symptômes mineurs qui peuvent facilement être attribués à une autre cause (21-23). Les hommes souffrant d'une infection gonococcique urétrale présentent habituellement des symptômes mais, pour les deux sexes, les infections rectales ou pharyngées sont le plus souvent asymptomatiques (24-26).

\section{La gonorrhée est hautement transmissible}

L'infection gonococcique se propage facilement. On évalue les taux de transmission à un taux allant de $50 \%$ à $60 \%$ à la suite d'un seul rapport sexuel entre un homme infecté et une femme non infectée et à $20 \%$ entre une femme infectée et un homme non infecté (27). Cette combinaison d'un organisme très contagieux et d'un manque de symptômes entraîne un taux élevé de transmission ultérieure.

\section{Les contacts sexuels pendant un voyage ne sont pas rares}

On a démontré que le voyage est associé à des comportements sexuels à risque (28-30). Les voyages sont souvent décrits comme une évasion temporaire pour se soustraire aux attentes sociales qui sont le lot de la vie quotidienne, ce qui contribue à donner au voyageur l'impression qu'il n'est ni vu ni connu et qui I'incite à agir d'une manière qui ne serait pas acceptable dans son pays d'origine (30-32). La prévalence des rapports sexuels occasionnels chez les voyageurs internationaux est estimée, selon les données regroupées sur la question, à un taux allant de $20 \%$ à $34 \%$ environ $(30,33)$. Les hommes gais, bisexuels ou qui ont des rapports sexuels avec d'autres hommes (HARSAH) ont deux à trois fois plus tendance à déclarer avoir fréquenté un nouveau partenaire sexuel pendant un séjour dans une destination outremer (34-36) et la proportion des hommes gais, bisexuels et HARSAH qui ont des relations sexuelles anales non protégées avec un partenaire occasionnel varie entre $22 \%$ et $60 \%(30)$.

Les rapports récents portant sur la nouvelle souche FC428 impliquée dans les cas canadiens d'infections gonococciques résistantes aux agents antimicrobiens qui sont liés aux voyages nous rappellent que certaines parties du monde, plus particulièrement l'Asie du Sud-Est, ont été témoins de l'émergence de nouvelles souches résistantes (multirésistantes et ultrarésistantes) qui constituent un risque pour les Canadiens.

\section{Les individus hésitent à avoir recours aux tests de dépistage ou à les proposer}

Malheureusement, pour un grand nombre de raisons, les individus peuvent ne pas vouloir faire appel au dépistage. Ils ont tendance à sous-estimer le risque personnel qu'elles courent. Ils peuvent avoir l'impression que ces infections transmissibles sexuellement ne sont pas graves, craindre devoir subir des procédures invasives ou se sentir gênés à l'idée de passer un examen génital. D'autres obstacles, tels que les attitudes perçues ou anticipées des professionnels de la santé et du personnel de la clinique, peuvent donner à ces personnes l'impression qu'elles sont jugées et victimes de discrimination $(37,38)$. On signale, par exemple, que seulement $49 \%$ à $70 \%$ des hommes gais, bisexuels et HARSAH ont divulgué aux médecins leur orientation sexuelle $(39,40)$. En dernier lieu, des barrières sociales s'érigent lorsque le patient craint d'être rejeté par ses pairs (stigmatisation) dès qu'ils sauront qu'il aura subi un test sur les infections transmissibles sexuellement (37).

Les professionnels de la santé peuvent ne pas offrir de tests de dépistage. Ceux qui ne traitent ou ne gèrent pas fréquemment les infections transmissibles sexuellement peuvent ignorer comment et quand il convient de faire le test de dépistage des ITS, et que faire en cas de résultat positif. Le malaise créé par le fait d'avoir à demander au patient ses antécédents sexuels et de procéder à un examen génital, et le manque de temps dont dispose le professionnel en raison d'autres priorités médicales comptent parmi les autres obstacles au test de dépistage des ITS qui ont été relevés (41-44).

\section{Les recommandations en matière de traitement sont complexes et peuvent changer}

Les recommandations en matière de traitement continuent à changer afin de s'ajuster aux profils de résistance qui évoluent constamment. Par exemple, en fonction des niveaux de résistance croissants, I'ASPC a modifié ses recommandations en matière de traitement de première intention pour adopter la thérapie combinée (c'est-à-dire ceftriaxone ou céfixime plus azithromycine) en 2013. En 2017, elle a émis une recommandation supplémentaire pour un traitement alternatif (gentamicine et azithromycine) pour les infections 
gonococciques (45). Ce ne sont pas tous les cliniciens qui sont au courant des recommandations les plus à jour; certains peuvent se fier à d'autres recommandations.

Les recommandations en matière de traitement sont par ailleurs complexes. Les médicaments et les doses recommandées peuvent varier selon le site de l'infection ou l'activité sexuelle de la personne atteinte, ce qui signifie que le traitement prescrit pour une infection génitale simple ne sera pas adéquat pour le traitement d'une infection pharyngée qui est plus difficile à traiter avec succès.

\section{Le Canada est moins bien équipé qu'avant pour assurer le suivi des infections de gonorrhée résistante}

L'utilisation du test d'amplification des acides nucléiques (TAAN) a été dans une grande mesure considérée comme un progrès dans le domaine du dépistage des gonocoques, surtout en raison de sa facilité d'utilisation (le test peut être réalisé avec de l'urine) et de sa sensibilité élevée - jusqu'à $100 \%$ dans certains cas. L'une des conséquences indésirables du TAAN est le nombre moins élevé de cultures obtenues et que les cultures sont en ce moment sollicitées pour le test de sensibilité aux agents antimicrobiens. En 2016, par exemple, des 23708 cas déclarés, seulement $19 \%$ ont été mis sous culture (46), ce qui signifie que les données directes sur la résistance aux antimicrobiens n'étaient disponibles que pour environ le cinquième de tous les cas d'infections gonococciques au Canada.

\section{Les souches résistantes ont un avantage concurrentiel par rapport aux souches non résistantes}

Les souches multirésistantes et ultrarésistantes de toute infection bactérienne peuvent se propager rapidement et continuellement car elles sont difficiles à traiter.

\section{Recommandations pour la prise de mesures}

À la lumière de l'émergence récente de nouvelles souches résistantes aux options de traitement de première ligne qui nous restent, les cliniciens et les professionnels de la santé publique de première ligne peuvent procéder de quatre façons.

\section{Normaliser et augmenter le dépistage et promouvoir les pratiques sexuelles plus sûres}

Les professionnels de la santé peuvent atténuer les réticences touchant le dépistage. Une stratégie importante consiste à normaliser et à offrir le dépistage des infections gonococciques et d'autres d'infections transmissibles sexuellement et par le sang (ITSS) - dans le cadre des soins médicaux courants. Utiliser le TAAN sur un échantillon d'urine à des fins de dépistage peut réduire les obstacles, car il s'agit d'une méthode moins invasive pour le patient et qui exige moins de temps pour le clinicien.

Communiquez davantage les risques et les avantages liés au dépistage des ITS et aux pratiques sexuelles plus sûres en réalisant une évaluation des risques et en offrant des tests de dépistage aux patients qui ont besoin de conseils en matière de contraception ou à ceux qui ont un nouveau partenaire. Bien que les jeunes adultes soient le plus à risque, les adultes d'âge moyen ou mûr peuvent également être à risque de contracter une ITS et profiteraient ainsi du dépistage. Mettez l'accent sur le besoin d'utiliser des condoms correctement et de façon consistante.

L'Association canadienne de santé publique a mis au point une excellente ressource sur les pratiques exemplaires lorsqu'il est question d'enjeux délicats concernant la santé sexuelle, la toxicomanie et les ITSS afin d'aider les fournisseurs à évaluer le risque et à éduquer les patients (47). Cette ressource peut réduire les obstacles liés au malaise ressenti lors de la discussion des comportements à risque. Une courte évaluation des risques peut servir à rapidement cerner ou éliminer les principaux facteurs de risque associés au risque accru de contracter des ITS. On devrait demander aux patients dont les antécédents actuels ou passés constituent un facteur de risque potentiel d'ITS d'expliquer leurs antécédents encore plus en détail.

\section{Recommandations concernant le dépistage}

Le dépistage devrait être offert en fonction du risque. Les principaux facteurs de risque pour une infection gonococcique sont :

- Des antécédents en matière d'ITS (y compris l'infection au VIH)

- Un partenaire ayant reçu un diagnostic d'infection gonococcique

- Les jeunes de moins de 25 ans qui sont sexuellement actifs (en raison de la surreprésentation de ce groupe d'âge)

- Des relations sexuelles non protégées

- Des partenaires multiples

- Être gai, bisexuel ou HARSAH

- Un nouveau partenaire sexuel à l'occasion d'un voyage

Le dépistage est particulièrement important au cours de la grossesse, car l'infection non traitée peut causer une grave maladie chez le nouveau-né. La Société canadienne de pédiatrie a émis une recommandation sur la prophylaxie de l'ophtalmie néonatale (48). Toutes les femmes enceintes à risque devraient subir un test de dépistage lors de leur première consultation prénatale ou au moment de l'accouchement si elles n'ont pas auparavant fait l'objet d'un dépistage. Chez les couples VIH sérodiscordants, la présence de gonorrhée, de chlamydia ou d'autres ITS chez l'un ou l'autre partenaire peut accroître le risque de transmission du VIH (18-20). 


\section{TAAN et culture chez les individus asymptomatiques}

Pour le dépistage des hommes asymptomatiques, il est préférable d'effectuer le TAAN à travers l'urine du premier jet (49). Pour le dépistage des femmes asymptomatiques, les frottis vaginaux sont préférables et peuvent être prélevés par la patiente. Des échantillons recueillis par écouvillonnage cervical pour le NAAT peuvent être soumis. Le TAAN de l'urine est idéal lorsqu'un examen pelvien n'est pas indiqué ou refusé.

En fonction des antécédents et de la situation clinique, il peut être approprié de prélever des échantillons de sites anatomiques multiples (p.ex. tous les sites à risques). La culture demeure le test de premier choix en ce qu'elle permet le dépistage d'infections extra-génitales (pharyngées et rectales) - le TAAN validé peut être utilisé s'il est impossible de procéder à la culture. Du fait que les infections pharyngées sont habituellement asymptomatiques, il est extrêmement important d'utiliser la culture pour effectuer le dépistage chez les patients qui ont des antécédents en matière de relations sexuelles orales.

\section{Offrir des conseils pour la préparation au voyage et prendre note des itinéraires de voyage}

Les fournisseurs de soins de santé se doivent de conseiller les voyageurs avant le départ sur la nécessité d'adopter des pratiques sexuelles sécuritaires. Selon la destination, il peut s'avérer utile de discuter en particulier du risque de contracter une infection gonococcique résistante aux antimicrobiens.

Lorsque le patient présente des symptômes d'une ITS ou une exposition possible à une ITS, I'évaluation doit comprendre ses itinéraires de voyage. Dans le cas où l'évaluation révèle une exposition durant des relations sexuelles non protégées au cours du voyage, il y a lieu de se faire très circonspect face à la possibilité d'une infection gonococcique résistante aux antimicrobiens et, plus particulièrement, d'une infection dont une souche résistante encore absente au Canada serait responsable.

\section{Accroître la fréquence de diagnostic et le suivi au moyen d'un contrôle de guérison}

Les cultures sont importantes pour le diagnostic de patients symptomatiques et essentielles à la collecte de renseignements sur les tests de résistance antimicrobienne. Il faut cependant remarquer que le TAAN est également important, car c'est le test diagnostique le plus sensible. Lorsqu'un patient présente des signes ou des symptômes qui correspondent à ceux de I'infection gonococcique, on peut se fier à la culture et au
TAAN menés conjointement. Ils permettent de faire des tests de sensibilité aux antimicrobiens et d'identifier les souches d'antimicrobiens, alors que l'utilisation extrêmement sensible du TAAN permet de réduire le nombre de diagnostics ambigus.

Des échantillons devront être prélevés pour tous les sites exposés. Si des patients symptomatiques reçoivent une thérapie empirique (c'est-à-dire avant que les résultats des tests ne soient connus), des spécimens devront être prélevés avant le début du traitement. Étant donné les taux élevés d'infections concomitantes, les spécimens devront être prélevés pour le diagnostic tant des infections gonococciques que des infections par la chlamydia (50).

\section{Test de la gonorrhée génito-urinaire}

Pour les hommes symptomatiques présentant des infections génito-urinaires, il y a lieu de procéder dès que possible à la culture et à un écouvillon urétral pour la coloration de gram. Lorsqu'un examen des cultures n'est pas disponible, le TAAN de I'urine peut être utilisé (51). Chez les femmes symptomatiques, on peut procéder à un frottis vaginal et à l'écouvillonnage cervical en vue de la culture et du TAAN. Non seulement I'utilisation de la culture est-elle importante mais elle est fortement recommandée, surtout dans certaines circonstances (évaluation de la maladie inflammatoire pelvienne et pendant la grossesse). Les écouvillons vaginaux et les échantillons d'urine conviennent au TAAN. Pour les patients présentant des symptômes urétraux, un écouvillon urétral pour la culture peut également être utilisé.

\section{Test de la gonorrhée extragénitale}

Pour les personnes actives sexuellement qui présentent des signes et des symptômes extragénitaux ou qui ont des antécédents en matière de relations sexuelles orales ou anales passives, des spécimens rectaux et pharyngés doivent être recueillis à des fins de dépistage. La culture demeure la méthode de dépistage préférée pour le diagnostic des infections extragénitales qui sont fréquemment asymptomatiques. Si cette méthode n'est pas disponible, vérifiez si votre laboratoire est un laboratoire intégré qui offre des services de validation de spécimens cliniques (rectaux et pharyngés) autres que le type de spécimen recommandé par le fabricant (urine). Le cas échéant, le TAAN « validé » peut être utilisé pour des spécimens extragénitaux.

\section{Suivi avec un contrôle de guérison}

Assurez le suivi des cultures pour un contrôle de guérison à tous les sites positifs de trois à sept jours après la fin du traitement. Si le TAAN est l'unique option pour le contrôle de guérison, utilisez ce test de deux à trois semaines après la fin du traitement afin d'éviter des résultats faux-positifs. 
Les contrôles de guérison sont particulièrement importants dans les cas suivants :

- Infections pharyngées

- Femmes enceintes

- Risque élevé de gonocoques résistants aux antimicrobiens (la maladie a été diagnostiquée chez le partenaire ou à la suite d'un voyage ou d'un rapport sexuel dans une région où la prévalence des gonocoques résistants aux antimicrobiens est élevée)

- Modes de traitement de rechange utilisés en l'absence du traitement de première intention (ceftriaxone) en raison de l'impossibilité de pratiquer la thérapie intraveineuse

- $\quad$ Symptômes persistants

La reprise du test de dépistage en post-traitement six mois plus tard est recommandée en raison des risques de réinfection.

\section{Fournir une thérapie combinée à jour aux patients et à leurs partenaires}

Administrez la thérapie combinée à tous les patients infectés par le gonocoque (52). L'utilisation de deux antimicrobiens ayant différents modes d'action pourrait améliorer l'efficacité du traitement et prévenir ou, du moins, retarder l'émergence des gonocoques et la propagation de leur résistance aux antimicrobiens. Afin de prévenir le développement de la résistance aux antimicrobiens, il est préférable d'éviter la monothérapie basée sur l'azithromycine, à raison de 2 grammes, à moins de n'avoir aucune autre option. Le traitement rapide et approprié des patients infectés et de tous les partenaires sexuels des 60 jours précédents est essentiel pour prévenir la propagation de l'infection. Les professionnels de la santé publique locaux peuvent aider à retracer et joindre les contacts si nécessaire.

Toutes les personnes doivent être traitées conformément aux recommandations courantes (45). Ces recommandations sont également disponibles et accessibles grâce à l'application mobile des Lignes directrices canadiennes sur les infections transmissibles sexuellement qui fournit un accès pratique et rapide à la version à jour des sections sur le diagnostic et la gestion des ITS qui figurent dans les Lignes directrices. Elles peuvent être téléchargées gratuitement sur des appareils Apple $^{\mathrm{MC}}$ et Android ${ }^{\mathrm{MC}}$ et on peut y avoir accès sur le site Web des Lignes directrices (4).

\section{Recommandations en matière de traitement}

La thérapie combinée recommandée varie en fonction du site de I'infection et de la probabilité que se développe une résistance. Les Lignes directrices, en particulier, font la différence entre les options de traitement pour les infections gonococciques anogénitales non-complexes et le traitement des infections pharyngées (chez tous les adultes) et des infections chez les hommes gais, bisexuels et HARSAH.

Tableau 1 : Les quatre principaux éléments nécessaires à la préservation des options concernant le traitement de première intention de la gonorrhée résistante aux antimicrobiens

\begin{tabular}{|c|c|}
\hline Mesures & Détails \\
\hline $\begin{array}{l}\text { Normaliser et amplifier le dépistage et promouvoir le } \\
\text { sécurisexe }\end{array}$ & $\begin{array}{l}\text { - Offrir le dépistage des ITS dans le cadre des soins médicaux courants afin de réduire les } \\
\text { obstacles au dépistage et la stigmatisation associée aux ITS, et discuter des risques d'ITS, } \\
\text { du sécurisexe et des bénéfices du dépistage } \\
\text { - Il faut prélever des échantillons sur tous les sites d'exposition, afin d'augmenter les } \\
\text { occasions de diagnostic et de fournir le traitement approprié }\end{array}$ \\
\hline $\begin{array}{l}\text { Prodiguer des conseils avant le départ en voyage } \\
\text { Inclure les antécédents de voyage dans votre } \\
\text { évaluation du risque }\end{array}$ & $\begin{array}{l}\text { - Conseiller les voyageurs sur l'importance d'adopter des pratiques sexuelles plus sûres lors } \\
\text { de leurs visites touristiques; il pourrait se révéler approprié en fonction de leur destination } \\
\text { de discuter de leurs risques de contracter une infection gonococcique résistante aux } \\
\text { antimicrobiens } \\
\text { - Dans le cas où l'évaluation révèle une exposition à des risques à la suite de relations } \\
\text { sexuelles non protégées pendant les voyages, il y a lieu de se faire très circonspect } \\
\text { face à la possibilité d'une infection gonococcique résistante aux antimicrobiens et, plus } \\
\text { particulièrement, d'une infection dont une souche résistante encore absente au Canada } \\
\text { serait responsable }\end{array}$ \\
\hline $\begin{array}{l}\text { Augmenter l'utilisation de cultures en vue de poser } \\
\text { un diagnostic et du test de guérison }\end{array}$ & $\begin{array}{l}\text { - Le TAAN est pratique et très sensible de sorte qu'il peut accroitre la capacité de poser un } \\
\text { diagnostic confirmant une infection gonococcique. La culture fournit des renseignements } \\
\text { sur les sensibilités antimicrobiennes avant le traitement et elle est essentielle à } \\
\text { l'amélioration de la surveillance par la santé publique des modèles et des tendances en } \\
\text { matière de résistance antimicrobienne } \\
\text { - Lorsque les symptômes correspondent à ceux d'une infection gonococcique, il est } \\
\text { extrêmement important d'envisager utiliser la culture de pair avec le TAAN }\end{array}$ \\
\hline $\begin{array}{l}\text { Fournir une thérapie combinée à jour pour les } \\
\text { patients et leurs contacts }\end{array}$ & $\begin{array}{l}\text { - En raison de la résistance antimicrobienne croissante, la thérapie combinée est la } \\
\text { norme en matière de soins : le choix de la thérapie combinée doit être guidé par le site } \\
\text { d'infection et les antécédents du patient. Les modèles de résistance aux antimicrobiens } \\
\text { peuvent afficher des variations régionales } \\
\text { - Consultez les Lignes directrices ou le guide des ITS dans votre région pour obtenir des } \\
\text { recommandations détaillées en matière de traitement } \\
\text { - Le traitement de tous les partenaires sexuels des } 60 \text { jours précédents est essentiel. Les } \\
\text { professionnels de la santé publique locaux peuvent aider à retracer et notifier ces contacts } \\
\text { si nécessaire }\end{array}$ \\
\hline
\end{tabular}


Consultez les conseils figurant dans les guides de santé publique locaux, provinciaux et territoriaux pour obtenir des détails sur les recommandations en matière de traitement (4). Les conseils des guides locaux, provinciaux et territoriaux doivent être suivis lorsque les options de traitement sont fondées sur les données locales, provinciales ou territoriales sur la résistance.

En cas de contre-indications des céphalosporines en raison d'une allergie ou d'une résistance, les Lignes directrices recommandent de faire appel aux modes de traitement de rechange qui comprennent la gentamicine (4).

Si l'on suspecte une infection persistante à la suite du traitement, il y aura lieu d'effectuer des tests portant sur la sensibilité tant à la culture qu'aux antimicrobiens afin de confirmer l'échec thérapeutique et d'évaluer les options de traitement efficaces et appropriées, ce qui justifie une consultation auprès d'un expert en maladies infectieuses.

Ces quatre recommandations clés concernant les mesures à prendre sont résumées dans le tableau 1.

\section{Discussion}

Les taux d'infections gonococciques et de résistance des gonocoques aux antimicrobiens sont en hausse, tant à l'échelle nationale qu'à l'échelle internationale, et ils représentent une sérieuse menace pour la santé publique. Les cliniciens et les professionnels de la santé publique de première ligne sont bien placés pour effectuer le dépistage proactif et traiter les patients dont les résultats aux tests de dépistage des gonocoques ou de résistance des gonocoques aux antimicrobiens sont positifs, et conseiller les personnes à risque, notamment sur les risques de contracter des ITS et les risques liés aux voyages. Les cultures sont nécessaires pour porter un diagnostic le cas échéant et pour évaluer l'efficacité d'un traitement, prévenir la transmission continue et assurer la surveillance efficace de la résistance antimicrobienne.

\section{Nouvelles initiatives nationales}

En plus des efforts déployés par les professionnels de première ligne, I'ASPC a mis en place plusieurs initiatives pour améliorer la compréhension des niveaux et des tendances actuels de résistance de la gonorrhée aux antimicrobiens au Canada et fournir de meilleures données probantes afin d'orienter I'élaboration de lignes directrices sur le traitement et les interventions de santé publique.

En 2013, le système de surveillance accrue de la résistance de la gonorrhée aux antimicrobiens a été lancé dans plusieurs régions. Ce système de surveillance accrue des données de laboratoire et de renseignements épidémiologiques recueille des renseignements sur les caractéristiques démographiques et cliniques, les comportements à risques, les foyers d'infection, la résistance et la sensibilité aux antimicrobiens, le typage génomique et les renseignements sur le traitement prescrit (53). Les données du traitement recueillies dans le cadre de ce programme en 2016 indiquent que la majorité des cas se sont vu prescrire le traitement privilégié ou un autre traitement proposé dans les Lignes directrices (4).

Afin d'apporter son soutien aux régions éloignées qui ne peuvent produire d'isolats de gonocoques en culture, le Laboratoire national de microbiologie a mis au point des technologies novatrices permettant le dépistage et la prédiction de la résistance antimicrobienne directement à partir des spécimens du TAAN (54-56). Même s'il est important de remarquer que ces tests ne peuvent remplacer le calcul de la concentration minimale inhibitrice (CMI), axée sur les cultures, ils peuvent aider à la surveillance en prédisant la sensibilité antimicrobienne à la céphalosporine, à la ciprofloxacine et à l'azithromycine, et ils peuvent, avec l'aide des méthodes d'identification moléculaire, contribuer à augmenter la compréhension des types de gonorrhée circulant dans une localité.

En dernier lieu, afin de soutenir la surveillance de modèles mondiaux de résistance du gonocoque aux agents antimicrobiens, I'ASPC participe aux efforts de collaboration internationale. Les données de surveillance nationale sur la résistance aux antimicrobiens sont soumises au rapport de I'Organisation mondiale de la Santé intitulé WHO Global STI Surveillance et au Global Antimicrobial Resistance Surveillance System (GLASS). Dans le but de normaliser la caractérisation des gènes associés à la résistance antimicrobienne du gonocoque, I'ASPC, en collaboration avec les chercheurs des Centers of Disease Control and Prevention (CDC) américains et des chercheurs britanniques, australiens et suédois, a mis au point NG-STAR, une séquence continue du modèle de typage de la résistance antimicrobienne à l'échelle moléculaire permettant d'assurer le suivi de la propagation des souches de N. gonorrhoeae dans le monde entier (57).

\section{Conclusion}

Des efforts sont menés en collaboration aux échelles locale, provinciale, territoriale et fédérale entre les cliniciens et les professionnels de la santé publique afin de prévenir, de cerner, de traiter et de surveiller les infections gonococciques et la résistance des gonocoques aux agents antimicrobiens au Canada. Le temps est venu de peaufiner nos efforts collectifs visant à empêcher les souches émergentes de gonocoques multirésistants et ultrarésistants de s'implanter au Canada.

\section{Déclaration des auteurs}

M. B. - Conceptualisation, méthodologie, administration de projet - ébauche originale, examen et révision

M. G. R. - Conceptualisation, méthodologie - ébauche originale, examen et révision

S. A. - ébauche originale, examen et révision

U. A. - ébauche originale, examen et révision

K. S. - Rédaction - examen et révision

I. M. - Rédaction - examen et révision 


\section{Conflit d'intérêts}

Aucun.

\section{Remerciements}

Les auteurs aimeraient remercier Oscar Niragira pour son soutien technique.

\section{Financement}

Le présent travail a été soutenu par l'Agence de la santé publique du Canada.

\section{Références}

1. World Health Organization, Human Reproduction Programme. Scientists warn that antibiotic-resistant gonorrhoea is on the on the rise. News Release. Geneva (CH): WHO; July 7, 2017. www.who.int/reproductivehealth/ topics/rtis/amr-gonorrhoea-on-the-rise/en/

2. Agence de la santé publique du Canada. Maladies à déclaration obligatoire en direct. Ottawa (ON): ASPC 2017 (modifié déc 2018). http://maladies.canada.ca/declarationobligatoire/graphiques?c=yl

3. Agence de la santé publique du Canada, Laboratoire national de microbiologie. Surveillance nationale de la sensibilité aux antimicrobiens de Neisseria gonorrhoeae Rapport sommaire annuel de 2016. Ottawa (ON): ASPC 2016 (modifié fév 2018; accédé déc 2018) https://www.canada. $\mathrm{ca} / \mathrm{fr} /$ sante-publique/services/publications/medicamentset-produits-sante/surveillance-nationale-sensibilite-auxantimicrobiens-neisseria-gonorrhoeae-rapport-sommaireannuel-2016.html

4. Agence de la santé publique du Canada. Lignes directrices canadiennes sur les infections transmissibles sexuellement. Ottawa (ON): ASPC 2013. (Accédé jan 2019) https://www. canada.ca/fr/sante-publique/services/maladies-infectieuses/ sante-sexuelle-infections-transmissibles-sexuellement/ lignes-directrices-canadiennes/infections-transmissiblessexuellement.html

5. Unemo M, Shafer WM. Antimicrobial resistance in Neisseria gonorrhoeae in the 21st century: past, evolution, and future. Clin Microbiol Rev 2014 Jul;27(3):587-613. DOl PubMed

6. Tapsall JW, Ndowa F, Lewis DA, Unemo M. Meeting the public health challenge of multidrug- and extensively drug-resistant Neisseria gonorrhoeae. Expert Rev Anti Infect Ther 2009 Sep;7(7):821-34. DOI PubMed

7. Martin I, Sawatzky P, Allen V, Lefebvre B, Hoang LM, Naidu P, Minion J, Van Caeseele P, Haldane D, Gad RR, Zahariadis G, Corriveau A, German G, Tomas K, Mulvey MR. Neisseria gonorrhoeae, bactérie multirésistante et ultrarésistante au Canada, de 2012 à 2016. Relevé des maladies transmissibles au Canada 2019;45(2/3):45-54.
8. Alirol E, Wi TE, Bala M, Bazzo ML, Chen XS, Deal C, Dillon JR, Kularatne R, Heim J, Hooft van Huijsduijnen R, Hook EW, Lahra MM, Lewis DA, Ndowa F, Shafer WM, Tayler L, Workowski K, Unemo M, Balasegaram M. Multidrug-resistant gonorrhea: A research and development roadmap to discover new medicines. PLoS Med 2017 Jul;14(7):e1002366. DOl PubMed

9. Allen VG, Mitterni L, Seah C, Rebbapragada A, Martin IE, Lee C, Siebert H, Towns L, Melano RG, Low DE. Neisseria gonorrhoeae treatment failure and susceptibility to cefixime in Toronto, Canada. JAMA 2013 Jan;309(2):163-70. https:// DOl PubMed

10. Singh $A E$, Gratrix J, Martin I, Friedman DS, Hoang L, Lester R, Metz G, Ogilvie G, Read R, Wong T. Gonorrhea treatment failures with oral and injectable expanded spectrum cephalosporin monotherapy vs dual therapy at 4 Canadian sexually transmitted infection clinics, 2010-2013. Sex Transm Dis 2015 Jun;42(6):331-6. DOI PubMed

11. Lefebvre B, Martin I, Demczuk W, Deshaies L, Michaud S, Labbé AC, Beaudoin MC, Longtin J. Ceftriaxone-resistant Neisseria gonorrhoeae isolated in North America. Emerg Infect Dis 2018;24(2): DOI PubMed

12. Lahra MM, Martin I, Demczuk W, Jennison AV, Lee KI, Nakayama SI, Lefebvre B, Longtin J, Ward A, Mulvey MR, Wi T, Ohnishi M, Whiley D. Cooperative Recognition of Internationally Disseminated Ceftriaxone-Resistant Neisseria gonorrhoeae Strain. Emerg Infect Dis 2018 Apr;24(4):735-40. DOI PubMed

13. Ponci T, Fouere $S$, Braille $A$, Camelena $F$, Agsous M, Bebear C, Kumanski S, Lot F, Mercier-Delarue S, Ngangro NN, Salmona M, Schnepf N, Timsit J, Unemo M, Bercot B. Multidrug-resistant Neisseria gonorrhoeae failing tratment with ceftriaxone and doxycycline in France, November 2017. Euro Surveill 2018;23(21):1800264. DOI PubMed

14. Golparian D, Rose L, Lynam A, Mohamed A, Bercot B, Ohnishi M, Crowley B, Unemo M. Multidrug resistance Neisseria gonorrhoeae isolate, belonging to the internationally spreading Japanese FC428 clone, with ceftriaxone resistance and intermediate resistance to azithromycin, Ireland, August 2018. Euro Surveill 2018;23(47):1800617. DOI PubMed

15. Public Health England. UK case of Neisseria gonorrhoeae with high-level resistance to azithromycin and resistance to ceftriaxone acquired abroad. Health Protection Report Volume 12, Number 11. 2018. https://www.gov.uk/ government/uploads/system/uploads/attachment_data/ file/694655/hpr1118_MDRGC.pdf

16. Australian Government. Department of Health. Multi-drug resistant gonorrhoea 2018. http://www.health.gov.au/ internet/main/publishing.nsf/Content/mr-yr18-deptdepot004.htm

17. European Centre for Disease Prevention and Control. Rapid Risk Assessment. Extensively drug-resistant (XDR) Neisseria gonorrhoeae in the United Kingdom and Australia, 7 May 2018. https://ecdc.europa.eu/en/publications-data/rapid- 
risk-assessment-extensively-drug-resistant-xdr-neisseriagonorrhoeae-united

18. Laga M, Manoka A, Kivuvu M, Malele B, Tuliza M, Nzila N, Goeman J, Behets F, Batter V, Alary M, Heyward WL, Ryder RW, Piot P. Non-ulcerative sexually transmitted diseases as risk factors for HIV-1 transmission in women: results from a cohort study. AIDS 1993 Jan;7(1):95-102. DOI PubMed

19. Johnson LF, Lewis DA. The effect of genital tract infections on HIV-1 shedding in the genital tract: a systematic review and meta-analysis. Sex Transm Dis 2008 Nov;35(11):946-59. DOI PubMed

20. Fleming DT, Wasserheit JN. From epidemiological synergy to public health policy and practice: the contribution of other sexually transmitted diseases to sexual transmission of HIV infection. Sex Transm Infect 1999 Feb;75(1):3-17. DOI PubMed

21. Hook EW 3rd, Handsfield HH. Sexually Transmitted Diseases. 4th ed. The McGraw-Hill Companies, 2008. Gonococcal Infections in the Adult; p. 627-45.

22. Mayor MT, Roett MA, Uduhiri KA. Diagnosis and management of gonococcal infections. Am Fam Physician 2012 Nov;86(10):931-8. PubMed

23. John J, Donald WH. Asymptomatic urethral gonorrhoea in men. Br J Vener Dis 1978 Oct;54(5):322-3. PubMed

24. Klein EJ, Fisher LS, Chow AW, Guze LB. Anorectal gonococcal infection. Ann Intern Med 1977 Mar;86(3):340-6. DOI PubMed

25. American Academy of Pediatrics, Committee on Infectious Diseases. Red book: 2012 Report of the Committee on Infectious Diseases. 29th ed. Elk Grove Village, IL: American Academy of Pediatrics; 2012. Gonococcal Infections; p. 336344.

26. Komaroff AL, Aronson MD, Pass TM, Ervin CT. Prevalence of pharyngeal gonorrhea in general medical patients with sore throats. Sex Transm Dis 1980 Jul-Sep;7(3):116-9. DOI PubMed

27. Ison C. Atlas of sexually transmitted diseases and AIDS. 4th ed. Netherlands: Elsevier; 2010. Gonorrhea; p. 24-39.

28. Benotsch EG, Martin AM, Espil FM, Nettles CD, Seal DW, Pinkerton SD. Internet use, recreational travel, and HIV risk behaviors in men who have sex with men. J Community Health 2011 Jun;36(3):398-405. DOI PubMed

29. Sundbeck M, Emmelin A, Mannheimer L, Miörner H, Agardh A. Sexual risk-taking during travel abroad - a cross-sectional survey among youth in Sweden. Travel Med Infect Dis 2016 May-Jun;14(3):233-41. DOI PubMed

30. Svensson P, Sundbeck M, Persson KI, Stafström M, Östergren PO, Mannheimer L, Agardh A. A meta-analysis and systematic literature review of factors associated with sexual risk-taking during international travel. Travel Med Infect Dis 2018 Jul - Aug;24:65-88. DOI PubMed

31. Matteelli A, Capone S. The Holy Grail of prevention of sexually transmitted infections in travellers. Sex Transm Infect 2016 Sep;92(6):405-6. DOI PubMed
32. Theocharous A, Zopiatis A, Philaretou AG. The social construction of male tourism deviance: the case of Agia Napa (AN), Cyprus. Sexuality \& Culture 2015;19(4):816-34. DOI

33. Vivancos R, Abubakar I, Hunter PR. Foreign travel, casual sex, and sexually transmitted infections: systematic review and meta-analysis. Int J Infect Dis 2010 Oct;14(10):e842-51. DOI PubMed

34. Clift SM, Forrest SP. Factors associated with gay men's sexual behaviours and risk on holiday. AIDS Care 1999 Jun;11(3):281-95. DOI PubMed

35. Mercer $\mathrm{CH}$, Fenton KA, Wellings $\mathrm{K}$, Copas AJ, Erens B, Johnson AM. Sex partner acquisition while overseas: results from a British national probability survey. Sex Transm Infect 2007 Dec;83(7):517-22. DOl PubMed

36. Whelan J, Belderok S, van den Hoek A, Sonder G. Unprotected casual sex equally common with local and Western partners among long-term Dutch travelers to (sub) tropical countries. Sex Transm Dis 2013 Oct;40(10):797-800. DOl PubMed

37. Denison HJ, Bromhead C, Grainger R, Dennison EM, Jutel A. Barriers to sexually transmitted infection testing in New Zealand: a qualitative study. Aust N Z J Public Health 2017 Aug;41(4):432-7. DOI PubMed

38. Tilson EC, Sanchez V, Ford CL, Smurzynski M, Leone PA, Fox KK, Irwin K, Miller WC. Barriers to asymptomatic screening and other STD services for adolescents and young adults: focus group discussions. BMC Public Health 2004 Jun;4(1):21. DOI PubMed

39. Petroll AE, Mosack KE. Physician awareness of sexual orientation and preventive health recommendations to men who have sex with men. Sex Transm Dis 2011 Jan;38(1):63-7. DOl PubMed

40. Agüero F, Masuet-Aumatell C, Morchon S, Ramon-Torrell JM. Men who have sex with men: A group of travellers with special needs. Travel Med Infect Dis 2018 Nov;S1477-8939(18):30384-3. DOI PubMed

41. Carter JW Jr, Hart-Cooper GD, Butler MO, Workowski KA, Hoover KW. Provider barriers prevent recommended sexually transmitted disease screening of HIV-infected men who have sex with men. Sex Transm Dis 2014 Feb;41(2):137-42. DOI PubMed

42. Barbee LA, Dhanireddy S, Tat SA, Marrazzo JM. Barriers to Bacterial Sexually Transmitted Infection Testing of HIV-Infected Men Who Have Sex With Men Engaged in HIV Primary Care. Sex Transm Dis 2015 Oct;42(10):590-4. DOI PubMed

43. Traversy GP, Austin T, Ha S, Timmerman K, Gale-Rowe M. Un aperçu des récentes données probantes sur les obstacles et les facteurs favorables au dépistage du virus de l'immunodéficience humaine. Relevé des maladies transmissibles au Canada 2015;41(12):445-63. DOI

44. Drainoni ML, Dekker D, Lee-Hood E, Boehmer U, Relf M. HIV medical care provider practices for reducing high-risk sexual 
behavior: results of a qualitative study. AIDS Patient Care STDS 2009 May;23(5):347-56. DOI PubMed

45. Agence de la santé publique du Canada. Traitement des infections gonococciques par suite du retrait du marché de la spectinomycine: Déclaration d'orientation pour les traitements de remplacement. Ottawa (ON): ASPC 2017. https://www.canada.ca/fr/sante-publique/services/ publications/maladies-et-affections/gonorrhee-traitementde-remplacement.html

46. Agence de la santé publique du Canada. Système canadien de surveillance de la résistance aux antimicrobiens - Mise à jour 2018 : Sommaire. Ottawa (ON): ASPC 2018. (Accédé déc 2018). https://www.canada.ca/fr/sante-publique/ services/publications/medicaments-et-produits-sante/ systeme-canadien-surveillance-resistance-antimicrobiens2018-rapport-resume.html

47. Association canadienne de santé publique. Comment discuter de santé sexuelle, de consommation de substances et d'ITSS, un guide pour les dispensateurs de services. Ottawa (ON): ACSP 2017. https://www.cpha.ca/sites/default/ files/uploads/resources/stbbi/discussionguide_f.pdf

48. Moore DL, MacDonald NE; Canadian Paediatric Society, Infectious Diseases and Immunization Committee. Preventing ophthalmia neonatorum. Paediatr Child Health 2015 Mar;20(2):93-6. PubMed

49. Papp JR, Schachter J, Gaydos CA, Van Der Pol B; Centers for Disease Control and Prevention. Recommendations for the laboratory-based detection of Chlamydia trachomatis and Neisseria gonorrhoeae--2014. MMWR Recomm Rep 2014 Mar;63 RR-02:1-19. www.cdc.gov/mmwr/preview/ mmwrhtml/rr6302a1.htm PubMed

50. Lyss SB, Kamb ML, Peterman TA, Moran JS, Newman DR, Bolan G, Douglas Jr JM, latesta M, Malotte CK, Zenilman JM, Ehret J, Gaydos C, Newhall WJ; Project RESPECT Study Group. Chlamydia trachomatis among patients infected with and treated for Neisseria gonorrhoeae in sexually transmitted disease clinics in the United States. Ann Intern Med 2003 Aug;139(3):178-85. DOl PubMed

51. Davies PO, Low N, Ison CA. The role of effective diagnosis for the control of gonorrhoea in high prevalence populations. Int J STD AIDS 1998 Aug;9(8):435-43. DOI PubMed
52. Papp J, Trees D, Shapiro SJ; Centers for Disease Control, Division of STD Prevention, National Center for HIV/AIDS, Viral Hepatitis, STD, and TB Prevention. Cephalosporin-Resistant Neisseria Gonorrhoeae Public Health Response Plan. CDC; August 2012. https://www.cdc. gov/std/treatment/ceph-r-responseplanjuly30-2012.pdf

53. Agence de la santé publique du Canada. Rapport sur le système de surveillance accrue de la résistance de la gonorrhée aux antimicrobiens - Résultats de la phase pilote de 2014. Ottawa (ON): ASPC 2014 (Modifié jan 2018; accédé déc 2018) https://www.canada.ca/fr/sante-publique/services/ publications/maladies-et-affections/gonorrhee-2014-pilotesurveillance-resistance-antimicrobiens.html

54. Peterson SW, Martin I, Demczuk W, Bharat A, Hoang L, Wylie J, Allen V, Lefebvre B, Tyrrell G, Horsman G, Haldane D, Garceau R, Wong T, Mulvey MR. Molecular Assay for Detection of Ciprofloxacin Resistance in Neisseria gonorrhoeae Isolates from Cultures and Clinical Nucleic Acid Amplification Test Specimens. J Clin Microbiol 2015 Nov;53(11):3606-8. DOI PubMed

55. Peterson SW, Martin I, Demczuk W, Bharat A, Hoang L, Wylie J, Tyrrell G, Horsman G, Haldane D, Garceau R, Wong T, Mulvey MR. Molecular Assay for Detection of Genetic Markers Associated with Decreased Susceptibility to Cephalosporins in Neisseria gonorrhoeae. J Clin Microbiol 2015 Jul;53(7):2042-8. DOI PubMed

56. Peterson SW, Martin I, Demczuk W, Hoang L, Wylie J, Lefebvre B, Labbé AC, Naidu P, Haldane D, Mulvey MR. A Comparison of Real-Time Polymerase Chain Reaction Assays for the Detection of Antimicrobial Resistance Markers and Sequence Typing From Clinical Nucleic Acid Amplification Test Samples and Matched Neisseria gonorrhoeae Culture. Sex Transm Dis 2018 Feb;45(2):92-5. DOI PubMed

57. Demczuk W, Sidhu S, Unemo M, Whiley DM, Allen VG, Dillon JR, Cole M, Seah C, Trembizki E, Trees DL, Kersh EN, Abrams AJ, de Vries HJC, van Dam AP, Medina I, Bharat A, Mulvey MR, Van Domselaar G, Martin I. Neisseria gonorrhoeae Sequence Typing for Antimicrobial Resistance, a Novel Antimicrobial Resistance Multilocus Typing Scheme for Tracking Global Dissemination of N. gonorrhoeae Strains. J Clin Microbiol 2017 May;55(5):1454-68. Epub 2017 Feb 22. DOI PubMed 